\title{
Educación ambiental para el cumplimiento de los objetivos del Ejército Nacional ${ }^{1}$
}

\author{
https://doi.org/10.21830/9789585318342.03
}

Diana Carolina Contreras Gutierrez ${ }^{2}$

Escuela de Aviación del Ejército

Ingrid Yuliana Arango Calderón ${ }^{3}$

Escuela Militar de Cadetes "General José María Córdova”

\section{Resumen}

Las Fuerzas Armadas deben velar en todo el territorio nacional por la protección y defensa del medioambiente y los recursos naturales renovables (Congreso de la República de Colombia, 1993). Como estrategia, el Ejército Nacional ha implementado diferentes planes dentro de su política, además, ha direccionado la investigación para dar soluciones científico-tecnológicas a los problemas relacionados con el área ambiental. El principal objetivo de esta investigación es diagnosticar el estado actual del panorama ambiental en el Ejército Nacional y de las capacidades de investigación y desarrollo tecnológico en el área ambiental. Todo ello, mediante una investigación de tipo descriptiva, en la cual se realizó una priorización, identificando los ejes y objetivos en el área ambiental por parte del Ejército Nacional, con el fin de realizar un direccionamiento, a través de las líneas de investigación en el sistema de educación, para así medir las capacidades de ciencia y tecnología. Se concluye que existe una estrecha relación entre la protección del medioambiente y la seguridad nacional. Para lograr el cumplimiento de los objetivos del Ejército Nacional deben fortalecerse las capacidades y aumentar los proyectos que apuntan a las líneas de investigación del área medioambiente y biodiversidad.

Palabras clave: educación; investigación; medioambiente; militar.

1 Este capítulo hace parte de los resultados del proyecto de investigación "Estrategias didácticas basadas en la inteligencia emocional como herramienta para la formación del carácter de los estudiantes" del Grupo de Investigación en Ciencias Militares, de la Escuela Militar de Cadetes "General José María Córdova", registrado con el código COL0082556 y categorizado en C por Minciencias. Los puntos de vista y los resultados de este capítulo pertenecen a los autores y no reflejan necesariamente los de las instituciones participantes.

2 Magíster en Desarrollo y Gerencia de Proyectos (Escuela Colombiana de Ingeniería). Especialista en Formulación y Evaluación de Proyectos (Universidad Católica de Colombia). Administradora Ambiental (Universidad Distrital Francisco José de Caldas). Investigadora de la Escuela de Aviación del Ejército Nacional. https://orcid.org/0000-0002-8996-5348 - Contacto: dianacontrerasgutierrez@cedoc.edu.co

3 Capitán en el Ejército Nacional de Colombia. Especialista en Ciencias Militares para el Planeamiento (Centro de Educación Militar). Profesional en Ciencias Militares y Profesional en Administración Logística (Escuela Militar de Cadetes "General José María Córdova”). Investigadora de la Escuela Militar de Cadetes "General José María Córdova”, grupo de investigación en Ciencias Militares. Orcid: https://orcid.org/0000-00025995-9262 - Contacto: ingridarangocalderon@cedoc.edu.co 


\section{Introducción}

La seguridad nacional debe ser contemplada en tres aspectos, seguridad humana, seguridad social y seguridad ambiental (Vinola \& Padma, 2015). Por tal razón, es necesario ampliar la seguridad nacional con las nuevas amenazas que se vislumbran desde un enfoque ambiental, a partir de los nuevos desafíos que degradan al medioambiente y producen el cambio climático (O’Toole, 2017).

Para Alavi, Mirehei y Ahmadi (2017) existe una relación significativa entre el desarrollo sostenible de un país y la seguridad nacional. En otras palabras, la seguridad nacional mejora el entorno ecológico de un país (Kim \& Mesquita, 2015). Dabelko y Rogers (2016) identificaron diferentes ventajas ambientales que surgen de la cooperación militar, con fines de seguridad, de hecho, O’Toole (2017) afirma que en los países de Latinoamérica el medioambiente está siendo asegurado por las Fuerzas Militares.

Por otro lado, es importante resaltar que en los conflictos armados se ha causado un daño al medioambiente y los recursos naturales, en áreas de importancia ambiental como parques nacionales y áreas especiales de conservación (Smith, 2019; Kim \& Mesquita, 2015). De igual forma, un sin número de actividades de las Fuerzas Militares también han originado diferentes impactos en el medioambiente (Magagula, 2020; Petre et al., 2016). En consecuencia, el sector defensa de cualquier país está llamado a diseñar programas para reducir el riesgo de degradación del medioambiente y, a la vez, garantizar la utilización sostenible los recursos naturales (Magagula, 2020)

\section{Marco teórico}

\section{Panorama ambiental a nivel Colombia}

La parte ambiental en el sector público en Colombia se formalizó con la Ley 99 de 1993, en la cual se crea el Ministerio del Medioambiente, se establece la gestión y conservación del medioambiente y se organiza el Sistema Nacional Ambiental (SINA). En cuanto la normatividad, cabe destacar la Ley 1930, de gestión integral de los páramos en Colombia; el Decreto 948 de 1995, sobre reglamento, protección y control de la calidad de aire; la Ley 430 de 1998, de residuos peligrosos; el Decreto 2105/83, sobre la potabilización y suministro del agua; el Decreto 2183/96, de licencias ambientales; y el Decreto 0901/97, sobre utilización del agua como receptor de vertimientos. 
Por otra parte, dentro de los organismos públicos del sector ambiental a nivel nacional, se tiene como principal entidad el Ministerio de Medioambiente, autoridad ambiental en Colombia, junto con la Autoridad Nacional de Licencias Ambientales (ANLA) y Parques Nacionales de Colombia. Dentro de las autoridades ambientales regionales, se encuentran las Corporaciones Autónomas Regionales; institutos públicos tales como: el Instituto de Hidrología, Meteorología y Estudios Ambientales (Ideam), el Instituto de Investigaciones Marinas y Costeras (Invemar) o el Instituto Humboldt, entre otros.

En Colombia, los problemas ambientales se hacen cada vez más complejos para la sociedad, lo que lleva a reflexionar sobre los efectos adversos que se puedan generar (Ramírez, 2015). La población que habita Colombia percibe la existencia de problemas ambientales, tales como la calidad del aire; la calidad del agua de ríos, arroyos y subterránea. Se puede establecer que las comunidades expuestas reconocen estar enfrentadas a una elevada contaminación y presentar preocupación ante el impacto y las consecuencias para la salud y la vida diaria (Robledo et al., 2017).

Entre los impactos ambientales a los que se enfrenta Colombia, se encuentran: afectaciones ambientales derivadas de actividades ganaderas, que causan daños a la vegetación y las fuentes hídricas (Giraldo et al., 2020); los plaguicidas, que resultan muy peligrosos a causa de los contaminantes orgánicos persistentes (COP), pues son altamente tóxicos y permanecen tanto en el medioambiente como en los tejidos de los seres vivos (García et al., 2015); la deforestación y la creciente pérdida de especies, debidas a las actividades antrópicas (Galeano \& Mancera, 2018); y, finalmente, la minería, que genera impactos ambientales negativos en el agua, afectando los páramos y el futuro del recurso hídrico (Tarazona \& Lugos, 2019).

\section{Panorama Ambiental en el Ejército Nacional}

La Ley 99 de 1993, en su artículo 103, determina el papel fundamental que tienen las Fuerzas Armadas en relación con la defensa del medioambiente:

Las Fuerzas Armadas velarán en todo el territorio nacional por la protección y defensa del medioambiente y los recursos naturales renovables y por el cumplimiento de las normas dictadas con el fin de proteger el patrimonio natural de la nación, como elemento integrante de la soberanía nacional. (Congreso de la República, 1993) 
Para el 2018 el sector defensa, a través de la política ambiental, pone a disposición del Estado colombiano las capacidades de la Fuerza Pública para mitigar los efectos del cambio climático, proteger los ecosistemas y el ambiente (Ministerio de Defensa Nacional, 2018). En ese mismo orden de ideas, el Plan Estratégico del Ministerio Defensa (2018-2022) define la contribución a la protección de los recursos naturales y del medioambiente como una de las áreas misionales del Sector Defensa y Seguridad, convirtiéndose en un objetivo estratégico alineado con el Plan Nacional de Desarrollo (Ministerio de Defensa Nacional, 2019).

En cuanto a la planeación estratégica del Ejército Nacional, el quinto objetivo es propuesto para apoyar la protección de los recursos naturales como activos estratégicos de la nación y la gestión de riesgos de desastres (Ejército Nacional de Colombia, 2019). Para el cumplimiento de este objetivo el Ejército Nacional cuenta, principalmente, con el Departamento de Ingenieros, que tiene la capacidad de emitir lineamientos y directrices en materia de gestión ambiental, con el fin de mitigar el impacto al medioambiente (Ejército Nacional de Colombia, 2020).

\section{Ejes estratégicos y objetivos para la protección del medioambiente}

Para el año 2019, el Ejército Nacional emite el Plan Artemisa, el cual tiene como misión la defensa de parques nacionales y áreas protegidas, para mitigar el daño al medioambiente, aportar a la sostenibilidad y a la protección de la biodiversidad y el agua, así como a la declaración de activos estratégicos de la nación (Ejército Nacional de Colombia, 2018). El Plan Artemisa cuenta con tres ejes estratégicos, en los cuales se plantean rutas mediante sus líneas de acción, con el fin de dar cumplimiento a siete objetivos. El Plan Artemisa se encuentra clasificado dentro de la organización como restringido.

En la tabla 1 se muestran los ejes y objetivos identificados para el direccionamiento de la investigación y desarrollo tecnológico por parte del Sistema de Educación del Ejército Nacional. Esta selección se realizó de acuerdo con las capacidades de los grupos de investigación que conforman el Sistema de Ciencia y Tecnología y la orientación propuesta desde la educación. 
Tabla 1. Ejes estratégicos y objetivos propuestos en el Plan Artemisa

\begin{tabular}{|c|c|}
\hline Ejes estratégicos & Objetivos \\
\hline $\begin{array}{l}\text { - Protección del Estado y sus recursos. } \\
\text { - Control institucional del territorio. } \\
\text { - Fortalecimiento institucional. }\end{array}$ & $\begin{array}{l}\text { - Desmantelar las organizaciones criminales } \\
\text { que depredan el medioambiente. } \\
\text { - Neutralizar estructuras que hacen } \\
\text { presencia en Parques Naturales Nacionales } \\
\text { (PNN) y áreas protegidas. } \\
\text { - Contribuir con el mantenimiento de la } \\
\text { integridad ecológica de los ecosistemas } \\
\text { para la perpetuación de las especies endé- } \\
\text { micas o amenazas. } \\
\text { - Denegar los espacios de protección } \\
\text { medioambiental a la criminalidad. }\end{array}$ \\
\hline
\end{tabular}

Nota: se omitieron aquellos ejes y objetivos que no se pueden direccionar a través de la investigación y el desarrollo tecnológico.

Fuente: elaboración propia, con datos obtenidos del Plan Artemisa

(Ejército Nacional de Colombia, 2018).

\section{Investigación ambiental en el Ejército Nacional desde la educación}

En la actualidad, se ha identificado la necesidad de incorporar competencias ambientales en la formación militar (Magagula, 2020; De Andrade, 2020). Esto, adaptando las habilidades profesionales con competencias ambientales, con el fin de atacar el problema de insostenibilidad asociado al hecho de que el daño al medioambiente está llegando a su límite (De Andrade, 2020)

Asimismo, se ha resaltado la generación de conocimiento científico y técnico que permita el uso, aprovechamiento sostenible y protección de los mares colombianos y otros ecosistemas estratégicos (Ministerio de Defensa Nacional, 2018). A este respecto, para Magagula (2020), un programa de investigación ambiental en el sector defensa tiene un importante potencial para influir en programas para la gestión ambiental. Por tal razón, los proyectos de investigación del sector defensa en esta área han generado un gran impacto positivo en la sostenibilidad ambiental, el cambio climático y la biodiversidad (Rice \& Keysar, 2017; Hutchinson et al., 2015; Jeong et al., 2015).

Por todo lo anterior, el Comando de Educación y Doctrina del Ejército Nacional (CEDOC) — como unidad encargada de orientar planes y políticas de doctrina, educación, instrucción, entrenamiento, ciencia y tecnología, para fortalecer el desarrollo del personal militar (Ejército Nacional de Colombia, 2016)_ direccionó los conocimientos, la investigación y la formación con las necesidades 
del Ejército, estableciendo las áreas y líneas de investigación desde la educación. Con ese derrotero, se funda el área de investigación de Medioambiente y Biodiversidad, alineada con cinco objetivos de desarrollo sostenible (que se pueden observar en la tabla 2), a la política de defensa y seguridad, a los objetivos estratégicos del Ejército y a su misionalidad (CEDOC, 2019).

A partir de esta área, se definieron las líneas de investigación (que se pueden observar en la tabla 2) que sirven para orientar a los grupos de investigación en lo referente a la solución de problemas ambientales en el contexto militar, además de ayudarles a cumplir con las metas y objetivos identificados en los planes y directrices del Ejército Nacional (específicamente el Plan Artemisa) y a los Objetivos de Desarrollo Sostenible (ODS).

Tabla 2. Direccionamiento de las líneas de investigación con los objetivos del Plan Artemisa y los ODS

\begin{tabular}{|c|c|c|c|}
\hline $\begin{array}{c}\text { Línea de } \\
\text { investigación }\end{array}$ & $\begin{array}{l}\text { Objetivos de la línea de } \\
\text { investigación }\end{array}$ & ODS & $\begin{array}{l}\text { Objetivo estratégico del } \\
\text { Plan Artemisa }\end{array}$ \\
\hline Minería ilegal & $\begin{array}{l}\text { Caracterizar los impactos } \\
\text { ambientales que ha generado } \\
\text { la minería ilegal. Proponer } \\
\text { estrategias para desman- } \\
\text { telar la minería ilegal en } \\
\text { todo el territorio nacional, } \\
\text { priorizando zonas prote- } \\
\text { gidas y parques nacionales. } \\
\text { Desarrollar técnicas para } \\
\text { bio-remediar los impactos } \\
\text { ambientales generados por la } \\
\text { minería ambiental. } \\
\text { Establecer procesos y procedi- } \\
\text { mientos que mitiguen, mini- } \\
\text { micen y prevengan impactos } \\
\text { ambientales producidos por la } \\
\text { minería legal e ilegal. }\end{array}$ & $\begin{array}{l}\text { Vida de ecosistemas } \\
\text { terrestres. } \\
\text { Salud y bienestar. } \\
\text { Agua limpia y } \\
\text { saneamiento. }\end{array}$ & $\begin{array}{l}\text { Desmantelar las organi- } \\
\text { zaciones criminales que } \\
\text { depredan el medioambiente. } \\
\text { Denegar los espacios de } \\
\text { protección medioambiental a } \\
\text { la criminalidad. }\end{array}$ \\
\hline Deforestación & $\begin{array}{l}\text { Identificar las zonas vulne- } \\
\text { rables y vulneradas por la } \\
\text { deforestación } \\
\text { por parte de las organiza- } \\
\text { ciones criminales. } \\
\text { Diseñar estrategias que } \\
\text { prevengan y mitiguen la } \\
\text { deforestación. }\end{array}$ & $\begin{array}{l}\text { Vida de ecosistemas } \\
\text { terrestres. } \\
\text { Salud y bienestar. } \\
\text { Acción por el } \\
\text { clima. }\end{array}$ & $\begin{array}{l}\text { Desmantelar las organi- } \\
\text { zaciones criminales que } \\
\text { depredan el medioambiente. } \\
\text { Denegar los espacios de } \\
\text { protección medioambiental a } \\
\text { la criminalidad. } \\
\text { Neutralizar estructuras que } \\
\text { hacen presencia en PNN y } \\
\text { áreas protegidas. }\end{array}$ \\
\hline
\end{tabular}




\begin{tabular}{|c|c|c|c|}
\hline $\begin{array}{c}\text { Línea de } \\
\text { investigación }\end{array}$ & $\begin{array}{l}\text { Objetivos de la línea de } \\
\text { investigación }\end{array}$ & ODS & $\begin{array}{l}\text { Objetivo estratégico del } \\
\text { Plan Artemisa }\end{array}$ \\
\hline $\begin{array}{l}\text { Producción } \\
\text { más limpia } \\
\text { y gestión } \\
\text { sostenible }\end{array}$ & $\begin{array}{l}\text { Caracterizar procesos y } \\
\text { procedimientos logísticos, } \\
\text { operacionales, industriales, } \\
\text { administrativos, entre otros, } \\
\text { del Ejército Nacional. } \\
\text { Identificar los impactos } \\
\text { ambientales que se generan } \\
\text { por las actividades que realiza } \\
\text { el Ejército Nacional. } \\
\text { Implementar estrategias y } \\
\text { técnicas de producción más } \\
\text { limpia y gestión sostenible } \\
\text { para mitigar, prevenir, } \\
\text { corregir o compensar los } \\
\text { impactos que se realicen } \\
\text { producto de las actividades } \\
\text { del Ejército Nacional. }\end{array}$ & $\begin{array}{l}\text { Acción por el } \\
\text { clima. } \\
\text { Producción y } \\
\text { consumo respon- } \\
\text { sables. } \\
\text { Ciudades y comu- } \\
\text { nidades sostenibles. } \\
\text { Energía asequible y } \\
\text { no contaminante. }\end{array}$ & $\begin{array}{l}\text { Contribuir con el manteni- } \\
\text { miento de la integridad ecoló- } \\
\text { gica de los ecosistemas, para } \\
\text { la conservación de las especies } \\
\text { endémicas y/o amenazas. }\end{array}$ \\
\hline $\begin{array}{l}\text { Conservación } \\
\text { ecológica y } \\
\text { protección de la } \\
\text { biodiversidad }\end{array}$ & $\begin{array}{l}\text { Diseñar políticas para la } \\
\text { protección del medioam- } \\
\text { biente y la biodiversidad. } \\
\text { Establecer mecanismos que } \\
\text { garanticen el desarrollo soste- } \\
\text { nible. } \\
\text { Establecer el principio de } \\
\text { prevención en las acciones } \\
\text { que genere el Ejército } \\
\text { Nacional y amenacen la } \\
\text { preservación de los recursos } \\
\text { naturales. } \\
\text { Contribuir a la protección y } \\
\text { mantenimiento de recursos } \\
\text { naturales, biodiversidad y } \\
\text { ecosistemas. }\end{array}$ & $\begin{array}{l}\text { Vida de ecosistemas } \\
\text { terrestres. } \\
\text { Acción por el } \\
\text { clima. }\end{array}$ & $\begin{array}{l}\text { Contribuir en el manteni- } \\
\text { miento de la integridad ecoló- } \\
\text { gica de los ecosistemas para la } \\
\text { conservación de las especies } \\
\text { endémicas y/o amenazas. }\end{array}$ \\
\hline
\end{tabular}

Fuente: elaboración propia, con datos obtenidos del documento de Áreas y Líneas (CEDOC, 2019), del Plan Artemisa (Ejército Nacional de Colombia, 2018) y los Objetivos de Desarrollo Sostenible en: https://www.undp.org/content/undp/es/home/sustainable-development-goals.html

A partir del direccionamiento planteado por el CEDOC, los grupos de investigación pertenecientes al Sistema de Ciencia y Tecnología del Ejército contribuyen con sus capacidades a cada una de las líneas de investigación (ver tabla 3). Con el objeto de acceder a recursos para la financiación de proyectos de investigación y desarrollo tecnológico, el CEDOC, a través de la Dirección de Ciencia y Tecnología DITEC, plantea los retos por medio de la formulación de una convo- 
catoria anual. Así, para las "líneas priorizadas", correspondientes a la convocatoria 2019, se plantearon los siguientes retos: lucha contra la explotación de recursos naturales; cambio climático; y aprovechamiento de recursos renovables aplicados a la institución militar. En la convocatoria 2020, dirigida a las capacidades requeridas para la fuerza, se plantearon necesidades en desarrollos logísticos para optimizar el uso de recursos naturales, en estrategias para mitigar el riesgo de desastres y en planes para prevenir y mitigar incendios forestales, entre otros temas.

Tabla 3. Capacidades de los grupos de investigación en el área de medioambiente y biodiversidad

\begin{tabular}{|c|c|c|}
\hline Grupo de investigación & $\begin{array}{l}\text { Capacidad: línea de } \\
\text { investigación del grupo }\end{array}$ & $\begin{array}{c}\text { Aporte con el área del } \\
\text { medioambiente y biodiversidad }\end{array}$ \\
\hline \multirow[t]{2}{*}{$\begin{array}{l}\text { Grupo de investigación } \\
\text { en ingeniería y simulación }\end{array}$} & $\begin{array}{l}\text { Procesos, materiales e insumos } \\
\text { en ingeniera civil y militar. }\end{array}$ & $\begin{array}{l}\text { Uso de recursos naturales de manera } \\
\text { más eficiente. }\end{array}$ \\
\hline & $\begin{array}{l}\text { Desarrollo de procesos y } \\
\text { sistemas de seguridad y } \\
\text { defensa. }\end{array}$ & $\begin{array}{l}\text { Procesos de seguridad y defensa que } \\
\text { permitan el cumplimiento del Plan } \\
\text { Artemisa. }\end{array}$ \\
\hline \multirow[t]{2}{*}{$\begin{array}{l}\text { Grupo de investigación } \\
\text { ingenieros militares }\end{array}$} & $\begin{array}{l}\text { Ingeniería civil y militar } \\
\text { aplicada al desarrollo de la } \\
\text { tecnología. }\end{array}$ & $\begin{array}{l}\text { Uso de recursos naturales de manera } \\
\text { más eficiente. }\end{array}$ \\
\hline & Gestión del riesgo de desastres. & $\begin{array}{l}\text { Mitigación de impactos ambientales } \\
\text { Conservación de ecosistemas }\end{array}$ \\
\hline $\begin{array}{l}\text { Grupo de investigación } \\
\text { en logística militar }\end{array}$ & Logística, civil y militar. & $\begin{array}{l}\text { Producción más limpia. } \\
\text { Uso de recursos naturales de manera } \\
\text { eficiente. }\end{array}$ \\
\hline $\begin{array}{l}\text { Grupo de investigación } \\
\text { en aviación militar }\end{array}$ & Innovación y sostenibilidad. & Gestión ambiental en la aviación. \\
\hline $\begin{array}{l}\text { Grupo de investigación } \\
\text { INTEMIL }\end{array}$ & Gestión logística militar. & $\begin{array}{l}\text { Producción más limpia. } \\
\text { Uso de recursos naturales de manera } \\
\text { eficiente. }\end{array}$ \\
\hline
\end{tabular}

Nota: los grupos de investigación con líneas de investigación en seguridad y defensa que aporten al Plan Artemisa también contribuyen a la protección del medioambiente. Otros grupos han iniciado investigaciones relacionadas con el área ambiental y la biodiversidad, sin consolidar alguna línea de investigación.

De acuerdo con las capacidades de los grupos de investigación y el direccionamiento a través de convocatorias, en la tabla 4 se pueden identificar los proyectos de investigación y desarrollo tecnológico que apuntan al área de investigación de medioambiente y biodiversidad, aportando así al cumplimiento del Plan Artemisa. 
Tabla 4. Proyectos de investigación y desarrollo tecnológico que aportan al área de medioambiente y biodiversidad

\begin{tabular}{|c|c|c|}
\hline Línea de investigación & Proyectos de investigación & Impacto \\
\hline Minería ilegal & $\begin{array}{l}\text { Proyecto de investigación Grupo } \\
\text { de investigación en ingeniería y } \\
\text { simulación. }\end{array}$ & $\begin{array}{l}\text { Control y reporte geográfico del } \\
\text { territorio. }\end{array}$ \\
\hline Deforestación & $\begin{array}{l}\text { Proyecto de investigación Grupo } \\
\text { de investigación en ingeniería y } \\
\text { simulación. }\end{array}$ & $\begin{array}{l}\text { Control y reporte geográfico del } \\
\text { territorio. }\end{array}$ \\
\hline \multirow[t]{3}{*}{$\begin{array}{l}\text { Producción más limpia } \\
\text { y gestión sostenible }\end{array}$} & $\begin{array}{l}\text { Proyectos de investigación Grupo } \\
\text { de investigación ingenieros mili- } \\
\text { tares. }\end{array}$ & $\begin{array}{l}\text { Conservación de recursos naturales. } \\
\text { Reducir el consumo de recursos. } \\
\text { Producción limpia y autososteni- } \\
\text { miento. }\end{array}$ \\
\hline & $\begin{array}{l}\text { Proyecto de investigación Grupo de } \\
\text { investigación en aviación militar. }\end{array}$ & $\begin{array}{l}\text { Reducción de la contaminación } \\
\text { ambiental. }\end{array}$ \\
\hline & $\begin{array}{l}\text { Proyecto de investigación } \\
\text { Grupo de investigación para la } \\
\text { capacitación militar. }\end{array}$ & $\begin{array}{l}\text { Autosostenimiento. } \\
\text { Reducir el consumo de recursos. }\end{array}$ \\
\hline $\begin{array}{l}\text { Conservación ecológica } \\
\text { y protección de la } \\
\text { biodiversidad }\end{array}$ & $\begin{array}{l}\text { Proyecto de investigación Grupo de } \\
\text { investigación ingenieros militares. }\end{array}$ & $\begin{array}{l}\text { Conservación y recuperación de } \\
\text { ecosistemas y zonas protegidas. }\end{array}$ \\
\hline
\end{tabular}

Durante los años 2019 y 2020 se han llevado a efecto seis proyectos de investigación y desarrollo tecnológico, para el cumplimiento de los objetivos estratégicos del Ejército y del Plan Artemisa. En cuanto a los productos científicos que se han producido a partir del área ambiental y de biodiversidad, en la tabla 5 se muestran aquellos que han generado nuevo conocimiento y desarrollo tecnológico durante los últimos cinco años. 
Tabla 5. Productos de generación de nuevo conocimiento y desarrollo tecnológico que apuntan al área ambiental

\begin{tabular}{ll}
\hline \multicolumn{1}{c}{ Tipo de producto } & \multicolumn{1}{c}{ Producto } \\
\hline $\begin{array}{l}\text { Artículos publicados } \\
\text { en revista indexada }\end{array}$ & $\begin{array}{l}\text { De la aplicabilidad de la gestión del riesgo a la reciente declaratoria de } \\
\text { emergencia sanitaria de Bogotá. } \\
\text { Definición de lineamientos para la formulación de determinantes de riesgo } \\
\text { para Corpoguavio (Colombia). } \\
\text { Nuevas formas para optimizar la gestión del riesgo desde la perspectiva de } \\
\text { territorios resilientes en Colombia. } \\
\text { Amenazas nucleares, biológicas y químicas, } \\
\text { una estrategia de manejo. }\end{array}$ \\
& $\begin{array}{l}\text { Unidad de suministro de energía para dispositivos portátiles. } \\
\text { Patentes }\end{array}$ \\
Prototipos & $\begin{array}{l}\text { Manual de gestión de riesgos ambientales para puestos avanzados de vigi- } \\
\text { Regulaciones y normasitil. }\end{array}$ \\
\hline
\end{tabular}

Nota: productos científicos de los últimos cinco años que apuntan al área de medioambiente y biodiversidad.

Los productos y resultados de investigación de los últimos cinco años se alinean, principalmente, con la gestión de riesgo y el desarrollo tecnológico capaces de reducir la generación de residuos sólidos. Cabe resaltar que el Grupo de investigación de ingenieros militares es el mayor aportante de estos productos. Sin embargo, a partir del año 2019, con la implementación del Plan Artemisa y la formulación del área de investigación en medioambiente y biodiversidad, se ha promovido el desarrollo de investigaciones dirigidas a trabajos afines a esta área de investigación. Como resultado, se han presentado varias propuestas de investigación de diferentes grupos en el área ambiental y el fortalecimiento de capacidades.

Por otro lado, grupos de investigación de las escuelas han trabajado durante este último año en fortalecer la investigación relacionada con medioambiente; tal es el caso del Grupo de investigación en aviación militar de la Escuela de Aviación y el Grupo de investigación para la capacitación miliar de la Escuela de Armas Combinadas del Ejército, los cuales han desarrollado actividades para la fortalecer estas capacidades, como el encuentro ambiental, la producción de cartillas y la conformación de semilleros de investigación.

\section{Conclusiones}

El Plan Artemisa, puesto en marcha por parte del Ejército Nacional, fortalece la seguridad ambiental al constituirse como eje transversal para la seguridad 
nacional, tal y como lo sustentan Vinola y Padma (2015). El cumplimiento de los objetivos del Plan Artemisa está estrechamente relacionado con la seguridad nacional y la protección de los recursos naturales. Garantizar el desarrollo sostenible y comprometerse con el uso eficiente de los recursos son tareas que lleva a cabo el Estado para proteger la soberanía del país. Lo que resalta la importancia fundamental que tienen en tales labores las Fuerzas Militares y evidencia la estrecha relación entre la seguridad y el área ambiental (Alavi et al., 2017).

El Plan Artemisa representa la materialización de los objetivos en el área ambiental propuestos por parte del Ejército Nacional. Es así como desde la educación se ha realizado una alineación estratégica para identificar las directrices de investigación que den cumplimiento a tales metas. Estas líneas apuntan a dos enfoques identificados en el estado del arte: el primero busca aumentar la seguridad para evitar la explotación de recursos naturales por parte de grupos al margen de la ley, tomando en cuenta los impactos negativos que sufre el medioambiente a causa de los conflictos armados (Smith, 2019; Kim \& Mesquita, 2015); el segundo enfoque busca prevenir, mitigar y compensar los impactos ambientales generados por las actividades de las Fuerzas Militares, ya que, como señala Magagula (2020), estas generan efectos en el medioambiente.

En los resultados presentados se puede evidenciar que los productos científicos de los grupos de investigación durante los últimos cinco años no representan un gran aporte para la protección del medioambiente, solo se fortalecen la gestión del riesgo y la producción tecnológica, y se reduce el consumo de materiales. A partir del año 2019, con la emisión del Plan Artemisa y la estipulación de ciertas áreas y líneas de investigación, se desarrollan convocatorias de investigación y desarrollo tecnológico enfocadas a alcanzar objetivos del Ejército en línea con el tema medioambiental, con esto se ha generado un aumento de proyectos de investigación con propósitos más pertinentes y oportunos para este campo de estudio. Además, se han desarrollado propuestas que buscan medir diversos impactos en el medioambiente relacionados, por ejemplo, con reducir el consumo de recursos naturales, generar producción más limpia, minimizar impactos ambientales, fortalecer el autosostenimiento, y la conservación de áreas protegidas. Asimismo, se evidencia la consolidación de capacidades en los grupos de investigación con respecto al enfoque ambiental.

El sistema de educación, a través de la investigación y el desarrollo tecnológico, ha generado un impacto positivo en el objetivo del Plan Artemisa que busca contribuir en el mantenimiento de la integridad ecológica de los ecosistemas, para la conservación de las especies endémicas y/o la contención de amenazas, 
específicamente en los ecosistemas de páramo, mediante investigaciones que provean la reproducción del frailejón. Además, han surgido diferentes proyectos que buscan reducir el consumo de agua, mitigando el impacto ambiental sobre este recurso hídrico. Otro objetivo del Plan Artemisa que se ha desarrollado, es el de lograr neutralizar estructuras armadas que hacen presencia en Parques Naturales Nacionales (PNN) y áreas protegidas, a través del desarrollo tecnológico de Sistemas de Información Geográfica (SIG) que sirvan como herramientas para llevar a efecto el control territorial.

De este modo, desde la educación que brinda el Ejército Nacional, resulta estratégico fortalecer el área ambiental para dar cumplimiento a la política nacional e institucional, con el fin de alcanzar las metas y objetivos propuestos por la institución, el Ministerio de Defensa y el Estado, para cumplir con la protección y defensa del medioambiente. Para esto, es necesario fortalecer las capacidades de los grupos de investigación y apoyar proyectos relacionados con el área ambiental y la biodiversidad, pues ya se ha evidenciado cómo las investigaciones de las Fuerzas Militares han generado beneficios para la protección y el cuidado del medioambiente (Rice \& Keysar, 2017; Hutchinson et al., 2015; Jeong et al., 2015).

\section{Referencias}

Alavi-Ghasvni, S., Mirehei, M., \& Ahmadi, S. (2017). Security and Regional Development in Iran Case Study: Khorasan Razavi Province. Geopolitics Quarterly, 13(1), 125-145. https://www. magiran.com/paper/1712212?lang=en

CEDOC. (2019). Áreas y líneas de investigación. CEDOC.

Congreso de la República de Colombia. (1993). Ley 99. Por la cual se crea el Ministerio del Medioambiente, se reordena el Sector Público encargado de la gestión y conservación del medioambiente y los recursos naturales renovables, se organiza el Sistema Nacional Ambiental (SINA) y se dictan otras disposiciones. http://www.humboldt.org.co/images/documentos/pdf/ Normativo/1993-12-22-ley-99-crea-el-sina-y-mma.pdf

Dabelko, G., \& Rogers, W. (2016). Military-to-Military Cooperation on the Environment and Natural Disasters: Engagement for peacebuilding. En C. Bruch, C. Muffett, \& S. Nichols (Eds.), Governance, Natural Resources and Post-Conflict Peacebuilding (p. 277). Routledge. https://doi.org/10.4324/9780203109793-13

De Andrade, H. (2020). Autoethnography (Military, Environment) as Transdisciplinarization in Anthropocene Times. Cultural Studies Critical Methodologies, 20(6), 535-538. https://doi. org/10.1177/1532708620912803

Ejército Nacional de Colombia. (2016). Misión centro de educación y doctrina. Ejército Nacional de Colombia. https://www.cedoc.mil.co/comando_educacion_doctrina/conozcanos/mision_393724

Ejército Nacional de Colombia. (2018). Artemisa Sostenimiento ambiental, preservación del agua, la biodiversidad y los recursos naturales, como activos estratégicos de la nación. 
Ejército Nacional de Colombia. (2019). Mapa Estratégico del Ejército Nacional. Ejército Nacional de Colombia. https://www.ejercito.mil.co/s_i_g/objetivos_institucionales_calidad_223721

Ejército Nacional de Colombia. (2020). Departamento de ingenieros militares. Ejército Nacional de Colombia. https://www.ejercito.mil.co/conozcanos/organigrama/jefatura_estado_mayor_ planeacion_253757/departamento_ingenieros_militares

Galeano, E., \& Mancera, N. (2018). Efectos de la deforestación sobre la diversidad y la estructura del ensamblaje de macroinvertebrados en cuatro quebradas Andinas en Colombia. Revista de Biología Tropical, 66(4), 1721-1740. https://doi.org/10.15517/rbt.v66i4.31397

García, C., García, J., \& Vaca, M. (2015). Valoración económica en salud y medioambiente del control de contaminantes orgánicos persistentes en Colombia. Revista de Salud Pública, 17(6), 951-960. https://doi.org/10.15446/rsap.v17n6.51717

Giraldo, L., Chará, J., Chará-Serna, A., \& Ramírez, Y. (2020). Restauración de corredores ribereños en paisajes ganaderos de la zona andina colombiana: efectos tempranos en el ambiente acuático. Revista de la Academia Colombiana de Ciencias Exactas, Físicas y Naturales, 44(171), 652-664.

Hutchinson, J., Jacquin, A., Hutchinson, S., \& Verbesselt, J. (2015). Monitoring Vegetation Change and Dynamics on U.S. Army Training Lands Using Satellite Image Time Series Analysis. Journal of Environmental Management, 150, 355-366.

Jeong, K., Hong, T., Ban, C., Koo, C., \& Park, H. (2015). Life Cycle Economic and Environmental Assessment for Establishing the Optimal Implementation Strategy of Rooftop Photovoltaic System in Military Facility. Journal of Cleaner Production, 104, 315-327. https://doi. org/10.1016/j.jclepro.2015.05.066

Kim, C., \& De Mesquita, B. (2015). Ecological Security and the Promotion of Peace: A DMZ Eco-peace Park. Korean Journal of Defense Analysis, 27(4), 539-557.

Magagula, H. (2020). Military Integrated Environmental Management Programme of the South African National Defence Force. South African Geographical Journal, 102(2), 170-189. https:// doi.org/10.1080/03736245.2019.1661873

Ministerio de Defensa Nacional. (2018). Politica ambiental del sector defensa Colombia. https://www. policia.gov.co/sites/default/files/cartilla_politica_sectorial_ambiental.pdf

Ministerio de Defensa Nacional. (2019). Plan Estratégico del Sector Defensa y Seguridad: Guía de Planeamiento Estratégico (2018-2022). https:/www.mindefensa.gov.co/irj/go/km/docs/ Mindefensa/Documentos/descargas/Sobre_el_Ministerio/Planeacion/Politicas/Guia_ Planeamiento_Estrategico_2018-2022.pdf

O’Toole, G. (2017). Environmental security in Latin America. Routledge. https://doi. org/10.4324/9781315529417

OECD. (2018). Manual de Frascati 2015: Guía para la recopilación y presentación de información sobre la investigación y el desarrollo experimental. OECD. https://doi.org/10.1787/9789264310681-es

Petre, R., Rotariu, T., Zecheru, T., Petrea, N., \& Băjenaru, S. (2016). Environmental Long-Term Impact on A Romanian Military Testing Range. Central European Journal of Energetic Materials, 13(1), 3-19. https://doi.org/10.22211/cejem/64961

Ramírez, O. (2015). Identificación de problemáticas ambientales en Colombia a partir de la percepción social de estudiantes universitarios localizados en diferentes zonas del país. Revista internacional de contaminación ambiental, 31(3), 293-310. http://www.scielo.org.mx/scielo.php?script=sci_ arttext\&pid=S0188-49992015000300009 
Rice, S., \& Keysar, E. (2017). NEPA Assessments for Large-Scale Renewable Energy Projects on Army Land: Best Practices and Lessons Learned. Environmental Practice, 19(4), 222-226. https://doi. org/10.1080/14660466.2017.1372542

Robledo, R., Agudelo, C., García, J., García, C., \& Osorio, S. (2017). Calidad de vida y ambiente en comunidades próximas a la actividad de minería industrial en Boyacá, Colombia. Revista de Salud Pública, 19(4), 511-518. https://https://doi.org/10.15446/rsap.V19n4.70324

Smith, T. (2019). Critical perspectives on environmental protection in non-international armed conflict: Developing the principles of distinction, proportionality and necessity. Leiden Journal of International Law, 32(4), 759-779. https://doi.org/10.1017/S0922156519000372Anitha Florence Vinola, F., \& Padma, G. (2015). A qualitative analysis on the risk determination and national security. International Journal of Applied Engineering Research, 10(2), 5227-5233.

CEDOC. (2019). Áreas y lineas de investigación CEDOC. Colombia.

Ejército Nacional de Colombia. (2016). Misión Centro de Educación y Doctrina. Retrieved from https://www.cedoc.mil.co/comando_educacion_doctrina/conozcanos/mision_393724

Ejército Nacional de Colombia. Artemisa Sostenimiento ambiental, preservación del agua, la biodiversidad y los recursos naturales, como activos estratégicos de la nación (2018).

Ejército Nacional de Colombia. (2020). Departamento de Ingenieros. Retrieved from https://www. ejercito.mil.co/conozcanos/organigrama/jefatura_estado_mayor_planeacion_253757/departamento_ingenieros_militares

Galeano-Rendón, E., \& Mancera-Rodríguez, N. J. (2018). Effects of deforestation on macroinvertebrate diversity and assemblage structure in four Andean creeks in Colombia | Efectos de la deforestación sobre la diversidad y la estructura del ensamblaje de macroinvertebrados en cuatro quebradas Andinas en Colomb. Revista de Biologia Tropical, 66(4), 1721-1740. https://doi. org/10.15517/rbt.v66i4.31397

García-Ubaque, C. A., García-Ubaque, J. C., \& Vaca-Bohórquez, M. L. (2015). Economic assessment in health and environment from control of persistent organic pollutants in Colombia | Valoración económica en salud y medio ambiente del control de contaminantes orgánicos persistentes en Colombia. Revista de Salud Publica, 17(6). https://oi.org/10.15446/rsap. v17n6.51717

Giraldo, L. P., Chará, J., Chará-Serna, A. M., \& Ramírez, Y. P. (2020). Restoration of riparian corridors in cattle ranching landscapes of the Colombian Andes: Early effects on the aquatic environment | Restauración de corredores ribereńos en paisajes ganaderos de la zona andina colombiana: Efectos tempranos en el ambiente ac. Revista de La Academia Colombiana de Ciencias Exactas, Fisicas y Naturales, 44(171), 652-664. https://doi.org/10.18257/raccefyn.1063

Ramírez Hernández, O. (2015). Identification of environmental problems in Colombia from the social perception of university students located in different parts of the country | Identificación de problemáticas ambientales en Colombia a partir de la percepción social de estudiantes univ. Revista Internacional de Contaminacion Ambiental, 31(3), 293-310.

Robledo-Martínez, R., Agudelo-Calderón, C. A., García-Ubaque, J. C., García-Ubaque, C. A., \& Osorio-García, S. D. (2017). Quality of life and environment in communities close to industrial mining activity in Boyacá, Colombia | Calidad de vida y ambiente en comunidades próximas a la actividad de minería industrial en Boyacá, Colombia. Revista de Salud Publica, 19(4), 511-518. https://doi.org/10.15446/rsap.V19n4.70324 
Tarazona, Á. A., \& Lugos, A. D. C. (2019). Thinking socio-environmental change: An approach to collective actions in defense of the páramo de Santurbán (Santander, Colombia) | Pensar el cambio socioambiental: Un acercamiento a las acciones colectivas por el páramo de Santurbán (Santander, Colombia. Revista Colombiana de Sociologia, 42(1), 157-175. https://doi. org/10.15446/rcs.v42n1.73070

Vinola, F., \& Padma, G. (2015). A Qualitative Analysis on the Risk Determination and National Security. International Journal of Applied Engineering Research, 10(2), 5227-5233. https://www. researchgate.net/publication/282955410_A_qualitative_analysis_on_the_risk_determination_ and_national_security 